\title{
Durable and sustained immune tolerance to ERT in Pompe disease with entrenched immune responses
}

\author{
Zoheb B. Kazi, ${ }^{1}$ Sean N. Prater, ${ }^{1}$ Joyce A. Kobori, ${ }^{2}$ David Viskochil, ${ }^{3}$ Carrie Bailey, ${ }^{3}$ Renuka Gera, ${ }^{4}$ \\ David W. Stockton, ${ }^{5}$ Paul McIntosh, ${ }^{6}$ Amy S. Rosenberg, ${ }^{7}$ and Priya S. Kishnani' \\ 'Division of Medical Genetics, Department of Pediatrics, Duke University Medical Center, Durham, North Carolina, USA. \\ ${ }^{2}$ Department of Genetics, Kaiser Permanente, San Jose, California, USA. ${ }^{3}$ Division of Medical Genetics, Department of \\ Pediatrics, University of Utah, Salt Lake City, Utah, USA. ${ }^{4}$ Department of Pediatrics/Human Development, Michigan \\ State University, East Lansing, Michigan, USA. ${ }^{5}$ Division of Cenetic, Genomic and Metabolic Disorders, Departments of \\ Pediatrics and Internal Medicine, Wayne State University and Children's Hospital of Michigan, Detroit, Michigan, USA. \\ ${ }^{6}$ School of Medicine, University of North Carolina at Chapel Hill, North Carolina, USA. 'Division of Therapeutic Proteins, \\ Office of Biotechnology Products, Center for Drug Evaluation and Research, United States Food and Drug Administration, \\ Bethesda, Maryland, USA.
}

Authorship note: ZBK and SNP are first coauthors.

Conflict of interest: DWS reports receiving research and grant support from Genzyme. DWS also receives honoraria and consulting fees from Cenzyme and is a member of the Pompe Disease Registry Advisory Board. PSK reports receiving research and grant support from Cenzyme. PSK also receives honoraria and consulting fees from Genzyme and is a member of the Pompe Disease and the Gaucher Disease Registry Advisory Boards. Duke University and the inventors of the method of treatment and precursors of the cell lines used to generate the enzyme (rhCAA) used commercially have received royalties pursuant to the University's policy on inventions, patents, and technology transfer. This potential conflict for Duke University has been resolved through monetization. PSK and SNP are coinventors on a patent related to the use of bortezomib in the context of antibody titers. This does not alter the authors' adherence to all The Journal of Clinical Investigation Insight policies on sharing data and materials.

Submitted: February 1, 2016

Accepted: June 9, 2016

Published: July 21, 2016

Reference information:

JCI Insight. 2016;1(11):e86821.

doi:10.1172/jci.insight.86821.
BACKGROUND. Enzyme replacement therapy (ERT) has prolonged survival and improved clinical outcomes in patients with infantile Pompe disease (IPD), a rapidly progressive neuromuscular disorder. Yet marked interindividual variability in response to ERT, primarily attributable to the development of antibodies to ERT, remains an ongoing challenge. Immune tolerance to ongoing ERT has yet to be described in the setting of an entrenched immune response.

METHODS. Three infantile Pompe patients who developed high and sustained rhCAA IgC antibody titers (HSAT) and received a bortezomib-based immune tolerance induction (ITI) regimen were included in the study and were followed longitudinally to monitor the long-term safety and efficacy. A trial to taper the ITI protocol was attempted to monitor if true immune tolerance was achieved.

RESULTS. Bortezomib-based ITI protocol was safely tolerated and led to a significant decline in rhCAA antibody titers with concomitant sustained clinical improvement. Two of the 3 IPD patients were successfully weaned off all ITI protocol medications and continue to maintain low/no antibody titers. ITI protocol was significantly tapered in the third IPD patient. B cell recovery was observed in all 3 IPD patients.

CONCLUSION. This is the first report to our knowledge on successful induction of long-term immune tolerance in patients with IPD and HSAT refractory to agents such as cyclophosphamide, rituximab, and methotrexate, based on an approach using the proteasome inhibitor bortezomib. As immune responses limit the efficacy and cost-effectiveness of therapy for many conditions, proteasome inhibitors may have new therapeutic applications.

FUNDING. This research was supported by a grant from the Genzyme Corporation, a Sanofi Company (Cambridge, Massachusetts, USA), and in part by the Lysosomal Disease Network, a part of NIH Rare Diseases Clinical Research Network (RDCRN).

\section{Introduction}

Pompe disease (OMIM no. 232300, glycogen storage disease type II) - an autosomal recessive, multisystem neuromuscular disorder - is the result of mutations in GAA gene (OMIM no. 606800), which encodes the lysosomal enzyme acid alpha-glucosidase (GAA). Reduced GAA activity results in the pathological accumulation of intralysosomal glycogen in various tissues, particularly cardiac and skeletal muscle. GAA gene mutations in infantile Pompe disease (IPD) result in markedly reduced or a complete lack of functional GAA. As a result, the natural history of untreated IPD unfolds rapidly, culminating in death secondary to cardiorespiratory failure within the first 2 years of life $(1,2)$. In 2006, recombinant human GAA (rhGAA) was approved as an enzyme replacement therapy (ERT) for IPD, leading to prolonged survival and marked improvement in 
Table 1. Patient demographics and GAA mutation data for the 3 patients with infantile Pompe disease. ${ }^{A}$

\begin{tabular}{|c|c|c|c|}
\hline & Patient 1 & Patient 2 & Patient 3 \\
\hline Race & Caucasian & Caucasian/African American & Caucasian \\
\hline Age at symptom onset & 3.0 months (2.8 CGA) & 2.0 months (1.1 CGA) & $<1.0$ month $(<-0.4$ CGA) \\
\hline Age at diagnosis & 5.0 months (4.8 CGA) & 4.0 months (3.1 CGA) & 3.5 years \\
\hline Age at ERT start & 6.0 months (5.8 CGA) & 4.5 months (3.6 CGA) & 3.6 years \\
\hline GAA mutation allele 2 & c. $2481+102 \_2646+31$ del & c.1654delC & c. $1655 T>C$ \\
\hline Amino acid change allele 1 & p.Cys103Gly & p.Arg854X & p.Leu552Pro \\
\hline Amino acid change allele 2 & p.Gly828_Asn882del & p.Leu552SerfsX26 & p.Leu552Pro \\
\hline
\end{tabular}

clinical outcomes (3-5). While the prognosis for patients with IPD on ERT has generally improved, there is still substantial individual variability in clinical responses. Initially, a cross-reactive immunologic material-negative (CRIM-negative) status emerged as a poor prognostic factor for patients with IPD on ERT (6). CRIM-negative patients, having no residual GAA protein, are particularly at risk of developing a deleterious immune response to ERT (7). Even though CRIM-positive patients have some - albeit reduced - GAA protein sufficient to confer immunological tolerance to ERT, a significant subset still mounts an immune response to ERT, leading to clinical decline following initial improvement (8). Hence, it was established that it is the development of high-sustained rhGAA IgG antibody titers (HSAT; defined as antibody titers $\geq 51,200$ more than once at or beyond 6 months on ERT) that is closely associated with clinical decline in patients with IPD (8).

Several unsuccessful attempts to date have been made in IPD and other conditions treated with a therapeutic protein to either achieve immune tolerance or mitigate the immune response, including increasing the dose of therapeutic protein and implementing various drug regimens (9). Subsequently, successful immune tolerance induction (ITI) to ERT in IPD was achieved with a short course of therapy using rituximab, methotrexate, and i.v. immunoglobulin (IVIG), when administered at or shortly prior to ERT initiation (i.e., in the ERT-naive setting) (10-12). In another series of IPD cases, ITI using rituximab and sirolimus or mycophenolate has been used (13). However, to implement successful ITI, it is necessary to identify patients who would otherwise mount HSAT preemptively. As the prediction of subset of CRIM-positive patients likely to mount HSAT is not currently possible, it cannot be determined which patients will have benefits that outweigh the risks of immunosuppression. Furthermore, CRIM status is often not determined prior to ERT initiation, putting these infants at high risk of mounting an immune response. There are 2 reported cases of IPD with HSAT on ERT in which immunomodulation with various combinations of cyclophosphamide, IVIG, plasmapheresis, increased doses of rhGAA, and rituximab failed to lower antibody titers and resulted in continued clinical decline $(9,14)$. In another case report of IPD, plasma exchange and rituximab was successful in lowering antibody titers; however, in this case, the rhGAA IgG antibody titers at the time of plasma exchange were 3,200 at 24 weeks after initiation of ERT, as opposed to HSAT in the 2 case reports where HSAT persisted, despite all treatment approaches attempted including plasmapheresis (15). We previously reported that the addition of the proteasome inhibitor bortezomib dramatically diminished HSAT in patients with clinical decline, presumably by targeting antibody-producing plasma cells, leading to marked clinical improvement in these 3 patients with otherwise terminal IPD (16). Here, we report the first successful achievement to our knowledge of true long-term immune tolerance to ongoing ERT in an entrenched immune response setting in 2 of these 3 cases and a significantly attenuated protocol resulting in low antibody titers in the third case. B cell recovery has been noted in all 3 cases. Addition of a proteasome inhibitor to disease-specific ITI protocols has potential therapeutic implications for other conditions attributable to or complicated by entrenched antibody responses.

Study design and patient characteristics. We included patients who met criteria for IPD (classic or atypical), treated with rhGAA infusions at cumulative doses of $20-40 \mathrm{mg} / \mathrm{kg}$ weekly or biweekly (17), who 


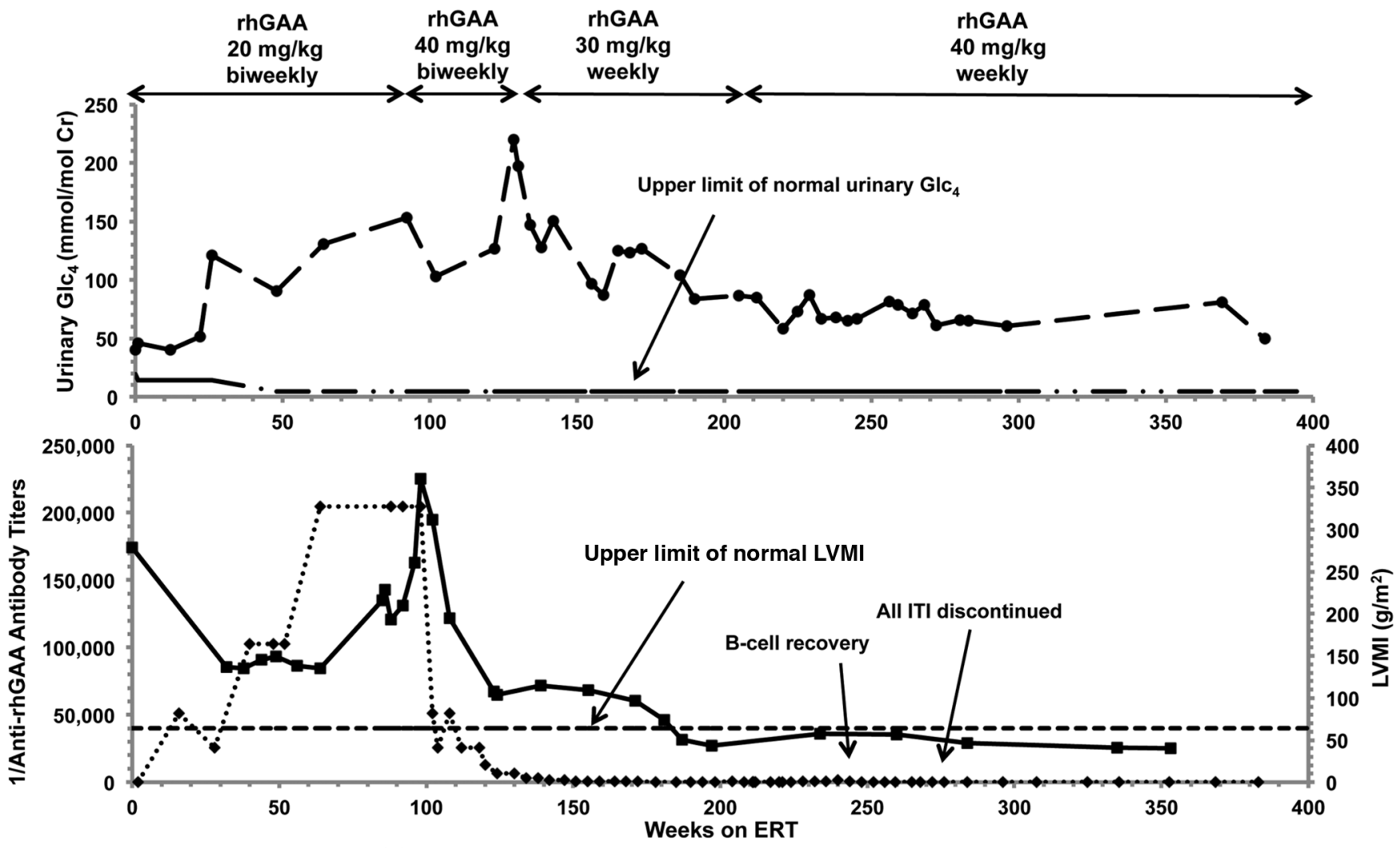

Cyclophosphamide $\left(250 \mathrm{mg} / \mathrm{m}^{2} \mathrm{IV}\right) \mathrm{Tt}$

\begin{abstract}
Rituximab (375 mg/m² IV)
Bortezomib $\left(1.3 \mathrm{mg} / \mathrm{m}^{2} \mathrm{IV}\right)$

Methotrexate (15 $\left.\mathrm{mg} / \mathrm{m}^{2} \mathrm{PO}\right)$

IVIG (400 - $500 \mathrm{mg} / \mathrm{kg}$ IV)
\end{abstract}

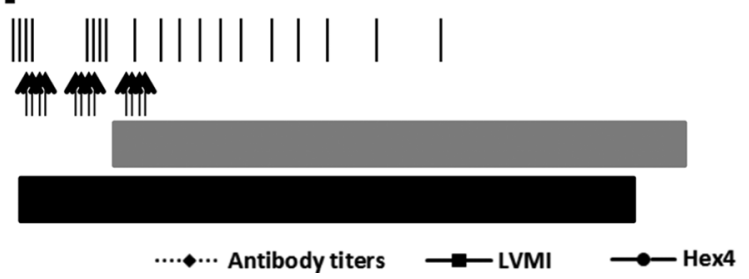

Figure 1. Dosing profile of recombinant human acid alpha-glucosidase (rhCAA) and immunomodulation, as well as rhGAA IgG antibody titers, left ventricular mass index ( $A$ and $B$; in $\mathrm{g} / \mathrm{m}^{2}$ ), and urinary glucose tetrasaccharide (in $\mathrm{mmol} / \mathrm{mol}$ creatinine) over time for Patient 1 . Glc $\mathrm{c}_{4}$, glucose tetrasaccharide; LVMI, left ventricular mass index; ERT, enzyme replacement therapy; IVIG, i.v. immunoglobulin. Upper limit of normal LVMI is $64 \mathrm{~g} / \mathrm{m}^{2}(18)$. Upper limit of normal urinary $\mathrm{Glc}_{4}$ for age < 6 months: $19 \mathrm{mmol} / \mathrm{mol}$ creatinine; age 6-12 months: $14 \mathrm{mmol} / \mathrm{mol}$ creatinine; age $\geq 1$ year: $4.4 \mathrm{mmol} / \mathrm{mol}$ of creatinine (19).

developed HSAT and had been administered a bortezomib-based ITI protocol as previously described (16). Written informed consent was provided by the respective parents/guardians for all patients under IRBapproved protocols. Clinical and laboratory data on patients 1, 2, and 3 from baseline through weeks 159, 229, and 174 of ERT, respectively, have been reported previously (16). Follow-up data for an additional 230, 204, and 189 weeks, respectively, are presented here. Medical records on these patients were reviewed up to October 2015; the time of their most recent follow-up assessment time point was determined based on the latest assessment available at or before October 1, 2015. Patient demographics and mutation data are shown in Table 1, and key clinical findings prior to the initiation of ERT (i.e. at baseline/week 0), initiation of bortezomib, and most recent follow-up are shown in Table 2 and Supplemental Table 1; supplemental material available online with this article; doi:10.1172/jci.insight.86821DS1.

\title{
Case reports
}

Patient 1. Patient 1 is an 8.0-year-old male of mixed European descent who presented at age 3 months with hypotonia and feeding difficulties. He was diagnosed with CRIM-positive IPD at age 5 months (see Table 1). At diagnosis, he had significant cardiomyopathy (left ventricular mass index [LVMI]: $278.6 \mathrm{~g} / \mathrm{m}^{2}$ ) and generalized hypotonia. At baseline, he did not require any respiratory support and received oral feeds. At age 6 months, he began biweekly rhGAA at $20 \mathrm{mg} / \mathrm{kg}$. Clinical improvement persisted for the first 8-9 months of 
Table 2. Key laboratory findings at enzyme replacement therapy start, bortezomib start, and at most recent follow-up at time of database lock for the 3 patients with infantile Pompe disease. ${ }^{A}$

\begin{tabular}{|c|c|c|c|}
\hline & Patient 1 & Patient 2 & Patient 3 \\
\hline Weeks on ERT at BTZ start & 99 & 154 & 88 \\
\hline LVMI at ERT start $\left(\mathrm{g} / \mathrm{m}^{2}\right)^{\mathrm{B}}$ (week 0$)$ & 278.6 & 200.9 & $\mathrm{WNL}^{\mathrm{C}}$ \\
\hline $\begin{array}{l}\text { LVMI at BTZ start }\left(\mathrm{g} / \mathrm{m}^{2}\right)^{\mathrm{B}} \\
\text { (time since ERT start) }\end{array}$ & 360.3 (week 98) & 137.0 (week 149) & WNL ${ }^{c}$ \\
\hline $\begin{array}{l}\text { Most recent LVMI }\left(\mathrm{g} / \mathrm{m}^{2}\right)^{\mathrm{B}} \\
\text { (time since ERT start) }\end{array}$ & 40 (week 353) & 28.92 (week 426) & $W_{N L^{c}}$ \\
\hline $\begin{array}{l}\text { Urinary } \mathrm{Clc}_{4} \text { level at } \mathrm{BTZ} \mathrm{start}^{\mathrm{D}} \\
\text { (mmol/mol creatinine) (time since ERT start) }\end{array}$ & 153.3 (week 92) & 41.9 (week 140) & 149.2 (week 90) \\
\hline $\begin{array}{l}\text { Most recent urinary } \mathrm{Glc}_{4} \text { level } \\
\text { (time since ERT start) }\end{array}$ & 49.7 (week 384) & 91.3 (week 348) & 74.8 (week 348) \\
\hline Most recent ITI status & $\begin{array}{l}\text { Off all ITI medications } \\
\text { since last } 114 \text { weeks }\end{array}$ & $\begin{array}{l}\text { Rituximab (yearly), } \\
\text { MTX (every other week), } \\
\text { IVIG (monthly) }\end{array}$ & $\begin{array}{l}\text { Off all ITI medications } \\
\text { since last } 163 \text { weeks }\end{array}$ \\
\hline Vaccination status & Up-to-date & $\begin{array}{l}\text { Up-to-date excluding } \\
\text { the live vaccines }\end{array}$ & Up-to-date \\
\hline \multicolumn{4}{|c|}{ 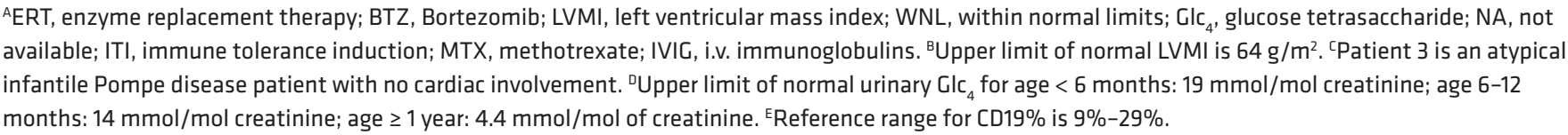 } \\
\hline
\end{tabular}

ERT, as evidenced by improved LVMI (135 g/m², week 38) and gross motor function (see Figure 1, Table 2, Supplemental Table 1, and ref. 16). However, he developed HSAT by week 48 and became ventilator dependent and required tube feeds by week 55. ERT antibody titers continued to increase, first peaking at 204,800 (week 64), which occurred with accelerated clinical decline and increased urinary Glc levels (39.9 $\mathrm{mmol} / \mathrm{mol}$ creatinine, week 0 , to $130.4 \mathrm{mmol} / \mathrm{mol}$ creatinine, week 64). Despite attempts at immunomodulation with cyclophosphamide and rituximab between weeks 86 and 95, titers remained unchanged at 204,800 from week 64-98. His cardiac status continued to decline as evidenced by an increase in LVMI $\left(360.3 \mathrm{~g} / \mathrm{m}^{2}\right.$, week 98$)$, resulting in a near-complete left ventricular cavity obstruction and significant left ventricular outflow tract obstruction. By week 98, he was unable to move his extremities, and voluntary motor function was limited to ocular movements only. A bortezomib-based regimen was started at week 99.

Patient 2. Patient 2 is an 8.7-year-old mixed European descent/African American male who presented at age 2 months with a cardiac murmur; echocardiogram showed significant biventricular hypertrophy. He was diagnosed with CRIM-negative IPD at age 4 months (see Table 1). At baseline, he had generalized hypotonia, tongue protrusion, facial myopathy, and cardiomegaly (LVMI: $200.9 \mathrm{~g} / \mathrm{m}^{2}$ ); however, he did not need any respiratory support and tolerated oral feeds. He initiated ERT at age 4.5 months at $20 \mathrm{mg} / \mathrm{kg}$ biweekly. He initially benefited clinically from ERT, as evidenced by reduced LVMI (95 g/m², week 27) and improved gross motor function (Table 2, Supplemental Table 1, Figure 2, and ref. 16). At week 43, he first developed HSAT, and his antibody titers continued to rise, peaking at 819,200 (week 87), accompanied by a plateau of his gross motor development and increased urinary Glc levels $(16.8 \mathrm{mmol} / \mathrm{mol}$ creatinine, week 43 , to 23.2 $\mathrm{mmol} / \mathrm{mol}$ creatinine, week 80 ). An ITI protocol was initiated at week 87 , with rituximab and methotrexate, resulting in a reduction in titers $(102,400$, week 115$)$. Initially, he maintained adequate physical activity and progressed in developmental motor milestones; an initial phase of improvement in LVMI $\left(100 \mathrm{~g} / \mathrm{m}^{2}\right.$, week 87 , to $62.3 \mathrm{~g} / \mathrm{m}^{2}$, week 103) was also noted, but the values deteriorated thereafter $\left(62.3 \mathrm{~g} / \mathrm{m}^{2}\right.$, week 103 , to $139 \mathrm{~g} / \mathrm{m}^{2}$, week 127). Antibody titers began to rise again and remained at 409,600 from week 143-151. At week 146, he was hospitalized for an upper respiratory tract infection, at which time he became ventilator dependent and required nasogastric tube feeds. His cardiac and motor status also deteriorated simultaneously and correlated with increased urinary $\mathrm{Glc}_{4}$ levels. A bortezomib-based regimen was started at week 154 . 


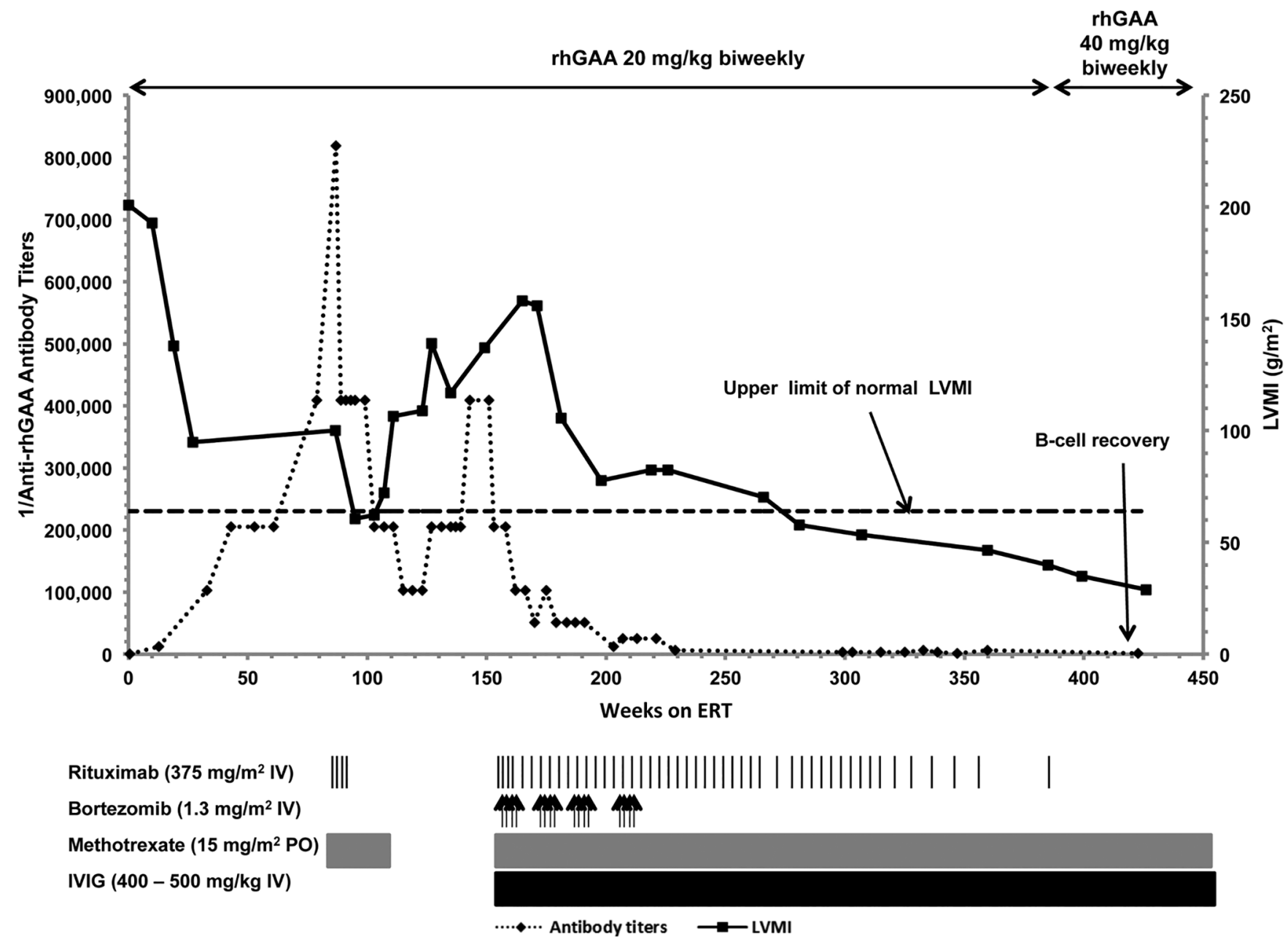

Figure 2. Dosing profile of recombinant human acid alpha-glucosidase (rhGAA) and immunomodulation, as well as rhGAA IgG antibody titers and left ventricular mass index $\left(\mathrm{g} / \mathrm{m}^{2}\right)$ over time for Patient 2. LVMI, left ventricular mass index; ERT, enzyme replacement therapy; IVIG, i.v. immunoglobulin. Upper limit of normal LVMI is $64 \mathrm{~g} / \mathrm{m}^{2}(18)$.

Patient 3. Patient 3 is a 10.5-year-old male of mixed European descent who presented with delayed motor milestones, inability to ambulate, and inability to sit unsupported. He was diagnosed with atypical CRIM-positive IPD at age 3.5 years (see Table 1). His age at symptom onset was $<1.0$ months. His electrocardiogram and echocardiogram were within normal limits, corroborating his atypical IPD diagnosis. Contrary to the other 2 classic IPD cases, he required ventilator support and gastrostomy tube feeds, at baseline. He also had an elevated baseline urinary $\mathrm{Glc}_{4}$ level $(67 \mathrm{mmol} / \mathrm{mol}$ creatinine). At age 3.6 years, he began receiving biweekly $20 \mathrm{mg} / \mathrm{kg}$ rhGAA. During the initial 34 weeks of treatment, his clinical status improved as he acquired new motor milestones and demonstrated an improved respiratory status (Table 2, Supplemental Table 1, Figure 3, and ref. 16). However, by week 56, he developed HSAT and reached his peak titer of 204,800 by week $64-90$. Along with the high titers, he experienced worsening of gross motor function and respiratory status. This was coincident with the rising urinary $\mathrm{Glc}_{4}$ levels, which peaked at week 90 (149.2 mmol/mol creatinine). A bortezomib-based regimen was started at week 88 .

\section{Results}

Bortezomib was administered twice weekly $\left(1.3 \mathrm{mg} / \mathrm{m}^{2}\right.$ of body surface area i.v. $)$ according to a standard dosing regimen (days $1,4,8$, and 11; equivalent to 1 cycle). Patients 1,2 , and 3 received a total of 3 , 4, and 6 cycles of bortezomib, respectively, along with rituximab, methotrexate, and IVIG (For complete details, see Table 2, Supplemental Table 1, Figures 1-3, and ref. 16). At the conclusion of the previous study, all 3 patients had experienced significant reduction in rhGAA IgG antibody titers, with the last titer measurement being 100 for Patient 1; 6,400 for Patient 2; and 3,200 for Patient 3, yet all remained on immunosuppressive 

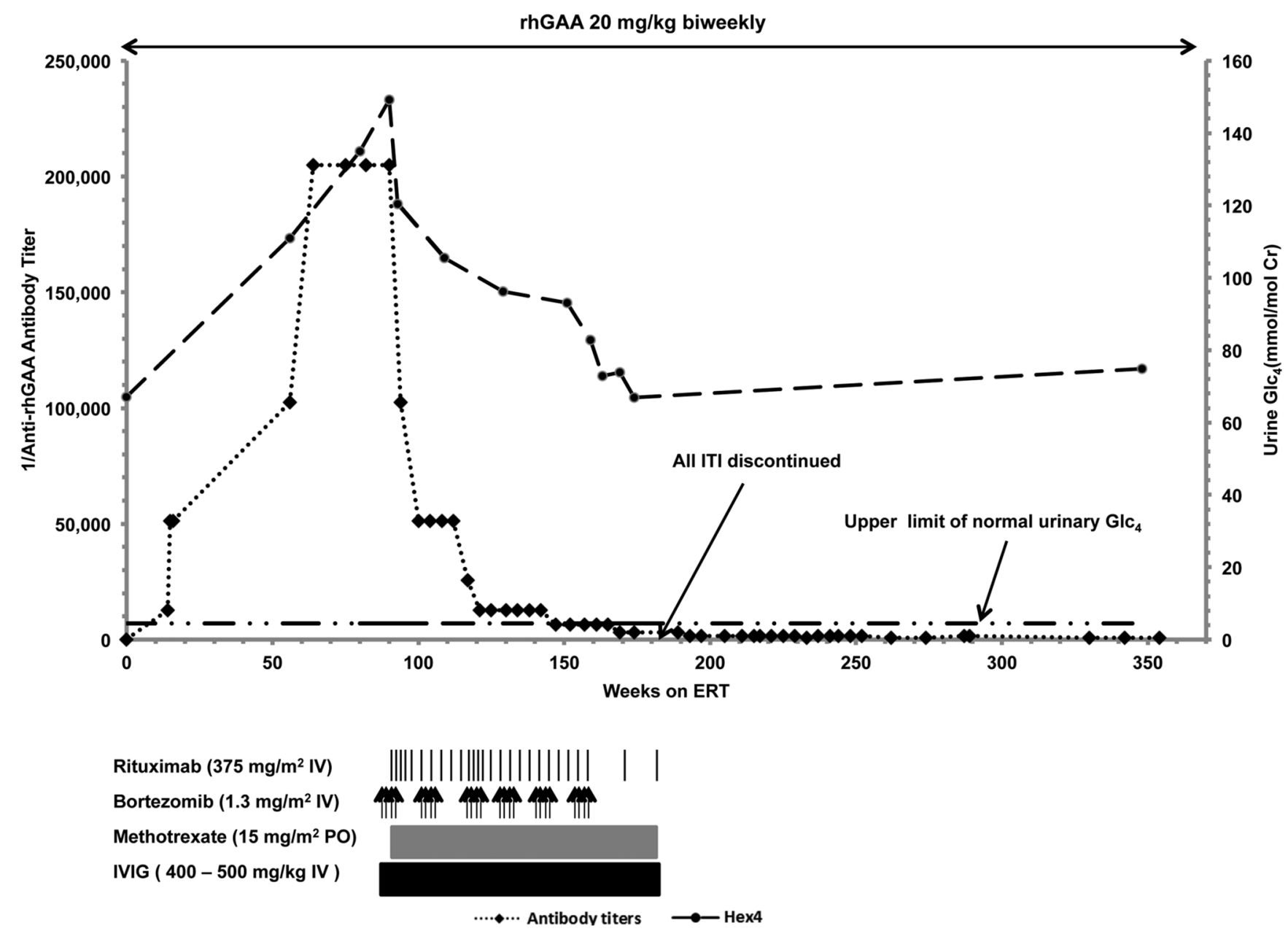

Figure 3. Dosing profile of recombinant human acid alpha-glucosidase (rhGAA) and immunomodulation, as well as urinary glucose tetrasaccharide (mmol/

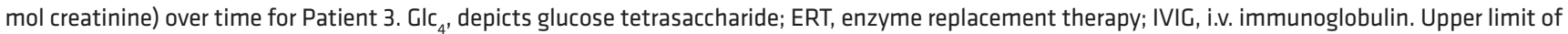
normal urinary Glc for age < 6 months: $19 \mathrm{mmol} / \mathrm{mol}$ creatinine; age 6-12 months: $14 \mathrm{mmol} / \mathrm{mol}$ creatinine; age $\geq 1$ year: $4.4 \mathrm{mmol} / \mathrm{mol}$ of creatinine (19).

therapy with no B cell recovery (as per CD19\%). At the conclusion of this study, all 3 patients continued to maintain low antibody titers, with their last titer measurement being 0, 1,600, and 800 at weeks 383, 423, and 354 for Patient 1, 2, and 3, respectively. The reduction of antibody titers was associated with continued clinical improvement. LVMI was significantly reduced to 72.0 and $82.5 \mathrm{~g} / \mathrm{m}^{2}$ for Patients 1 and 2 , respective$1 y$, at the conclusion of the previous study. No cardiomegaly was observed at any time point in Patient 3 , as this patient had atypical IPD. At the conclusion of this study, the LVMI for Patients 1 and 2 had normalized to 40.0 and $28.92 \mathrm{~g} / \mathrm{m}^{2}$ at weeks 353 and 426 , respectively (upper limit of normal LVMI is $64 \mathrm{~g} / \mathrm{m}^{2}$, ref. 18), and Patient 1 had a complete resolution of outflow tract obstruction. The urinary Glc $_{4}$ levels at weeks 159 , 227, and 174 (previous study end) were 87.1, 73.9, and $66.9 \mathrm{mmol} / \mathrm{mol}$ creatinine for Patients 1,2 , and 3, respectively, compared with 49.7, 91.3, and 74.8 at weeks 384,348 , and 348 on ERT, respectively (upper limit of normal urinary $\mathrm{Glc}_{4}$ for age $<6$ months: $19 \mathrm{mmol} / \mathrm{mol}$ creatinine; age $6-12$ months: $14 \mathrm{mmol} / \mathrm{mol}$ creatinine; age $\geq 1$ year: $4.4 \mathrm{mmol} / \mathrm{mol}$ of creatinine; ref. 19). It should be noted that Patient 3 did not have a dose increase and remained at $20 \mathrm{mg} / \mathrm{kg}$ biweekly, whereas Patient 1 is currently receiving $40 \mathrm{mg} / \mathrm{kg}$ weekly based on the treating physician's clinical decision. Patient 2 remained at $20 \mathrm{mg} / \mathrm{kg}$ biweekly for 439 weeks, after which the dose was increased to $40 \mathrm{mg} / \mathrm{kg}$ every other week. After the initiation of bortezomib and the decrease in antibody titers, respiratory status improved for all 3 patients, as reflected by decreased ventilator requirements. Moreover, feeding status, as assessed by their need of nasogastric (NG) tube or gastrostomy (G) tube feeds improved for Patients 2 and 3 (Supplemental Table 1).

After weeks 159, 227, and 174 - the previous study end for Patient 1, 2, and 3, respectively - no patient received additional bortezomib. However, all patients continued to receive additional methotrexate 
and rituximab, along with ERT. A trial to taper methotrexate and rituximab was attempted in all 3 patients, with Patients 1 and 3 successfully weaned off these medications at weeks 275 and 181, respectively. Patient 1 stopped receiving bortezomib at week 128, rituximab at week 207, and methotrexate at week 275. Patient 3 stopped receiving bortezomib at week 153, rituximab at week 181, and methotrexate at week 181. Patient 1 and 3 have been off all ITI since that time for an additional 114 weeks and 182 weeks, respectively, while continuing to receive ERT and maintaining low to no antibody titers. While Patient 2 is not completely off all ITI, he has been tapered significantly with respect to frequency of rituximab. His last dose of rituximab was on week 387. He had been off rituximab for 41 weeks when the data was collected for this study. He still receives methotrexate and IVIG based on the decision of the local treating physicians. Thus, 2 of 3 patients (Patients 1 and 3) are off all ITI medications; Patient 1, 2, and 3 have had full B cell recovery as per CD19\% of 10, 12, and 31 at weeks 245, 422, and 403, respectively. Patients 1 and 3 have been up-to-date on their vaccines, including live vaccines. For Patient 3 , the IgG titers against diphtheria vaccine was 0.4 IU of antitoxin/ $\mathrm{ml}$ and $\mathrm{IgG}$ titers against tetanus vaccine was $0.5 \mathrm{IU}$ of antitoxin $/ \mathrm{ml}$ at week 403 . Titers $>0.1$ $\mathrm{IU} / \mathrm{ml}$ have been correlated with protection against diphtheria and tetanus.

\section{Discussion}

Here, we report the first successful induction of long-term immune tolerance in the setting of an entrenched immune response to a lifesaving therapeutic protein. Like IPD, immunogenicity is known to limit the efficacy and cost-effectiveness of therapeutic proteins for many other conditions. IPD is inherently rapidly progressive, with long-term survival dependent on the continued efficacy of ERT. However, a significant percentage of patients with IPD develop deleterious, life-threatening sustained high-titer antibody responses to ERT that minimize or otherwise abolish its clinical efficacy. Given the existence of well-defined clinical outcome measures, these characteristics make IPD a useful model for readily assessing perhaps life-saving therapeutic approaches that have potentially broader clinical implications. Indeed, the 3 patients in this study initially improved clinically following commencement of ERT yet experienced subsequent clinical decline closely associated with HSAT development. While immune tolerance in IPD has been achieved in the ERT-naive setting using a combination of rituximab, methotrexate, and IVIG (20), no regimen to date to our knowledge has been able to induce immune tolerance once an entrenched immune response has developed.

Our previous study demonstrated that a plasma cell-targeting bortezomib-based regimen was well tolerated and was effective at dramatically reducing HSAT in 3 patients with terminal prognoses. However, at the end of the previous study, the patients were still receiving immune modulation, and an important question remained regarding the possible requirement of ongoing immune suppression over an indefinite period to maintain low antibody titers. In this report, we demonstrate a successful and life-saving induction of long-term immune tolerance in 2 of the 3 patients, and a marked decrease in use of immune suppression in the remaining case (Patient 2). All 3 have had a full recovery of their immune function, as shown by the recovery in CD19\%. Although Patient 2 currently remains on a maintenance regimen of infrequent, lowdose immunosuppressive drugs, we have successfully tapered these medications significantly in the face of sustaining low-antibody titers. All 3 continue to receive ERT, and most importantly, these patients continue to show improvement in previously lost motor milestones and show a resolution of cardiomyopathy.

The major dose-limiting toxicity of bortezomib, peripheral neuropathy, is a serious but usually reversible condition that is treatable via dose reduction or discontinuation of the drug (21). The 3 patients reported herein received bortezomib to hypothetically target the preexisting rhGAA antibody forming mature plasma cells, while rituximab eliminated the B cell population from which new plasma cells could be differentiated. Hence, there would be no need for ongoing bortezomib administration. The 3 patients, as we hypothesized, only required a few cycles of bortezomib. They have been followed closely for the possible development of side effects. However, no serious side effects, such as peripheral neuropathy, neuralgia, infections, have been noted with regard to treatment with the bortezomib-based regimen at any time point since its inception per reports from the treating physicians and caregivers. It however has to be acknowledged that this is a pediatric population with no formal assessments performed for peripheral neuropathy. Moreover, successful ITI, as seen in 2 of our 3 patients, and no need for additional bortezomib administration in all 3 patients precludes the need for chronic bortezomib administration, thereby mitigating the risk of the potential side effects.

Notably, clinician-investigators recently described successful adaptation of our bortezomib-based strategy to reduce preexisting high-antibody titers in a patient with mucopolysaccharidosis type II (MPS II) 
receiving idursulfase treatment, with their patient being on a maintenance immunomodulatory regimen at the end of their study (22). For cases as severe as Pompe, our protocol was well tolerated, safe, and effective in reducing a life-threatening immune response. Most importantly, our protocol was successful in inducing long-term immune tolerance. When this protocol was initiated, there was no knowledge in the field of how to successfully lower antibodies in the HSAT setting. This protocol was intended to be a rescue and to result in successful immune suppression. To ensure that there would be no recurrence of titers, rituximab was used repeatedly to keep B cells suppressed. Over time and with more experience, we started to taper the protocol and were successfully able to immune tolerize 2 patients and reduce the amount of immune suppressants significantly in the third patient. The 2 immune-tolerant cases, Patient 1 and 3 , had been off all immunomodulatory drugs for 114 weeks and 163 weeks, respectively, when the data were collected for this study and continue to receive ERT, while Patient 2 had been significantly weaned off the ITI protocol medications (Figure 2). The reduction in rhGAA IgG antibody titers with bortezomib-based ITI protocol provides evidence for our hypothesis that high sustained antibody responses are mediated by long-lived plasma cells, and it is only by targeting such populations that significant reductions in antibody titer occur and development of immune tolerance is feasible. We believe our immune-modulating strategy has potentially broader clinical implications. We realize the protocol administered to the earlier patients involved prolonged administration of rituximab; however, in the more recent cases receiving bortezomib-based protocol, the rituximab administration is significantly reduced (data not shown). This protocol has helped lower antibody titers in these terminally ill IPD patients; however, there is still a need for development of a less extensive protocol with agents that are antigen specific to reduce the immune response. Additional research will be needed to establish the proper dosing regimen of immune-suppressive agents in Pompe and other conditions and the optimal way to taper the maintenance regimen following bortezomib and/or other immunomodulatory agent treatments to ensure long-lasting tolerance.

\section{Methods}

IPD was diagnosed based on previously described methods (3). Analysis of GAA mutations, CRIM status, rhGAA IgG antibody titers (determined by Genzyme Corporation), LVMI (in $\mathrm{g} / \mathrm{m}^{2}$ ), and urinary glucose tetrasaccharide $\left(\mathrm{Glc}_{4} / \mathrm{Hex}_{4}\right.$, in $\mathrm{mmol} / \mathrm{mol}$ creatinine; a biomarker to monitor overall glycogen burden) were performed as previously described $(3,19,23,24)$. To monitor B cell recovery, flow cytometry was used to assess CD19\% using standard methods. Information regarding respiratory status, gross motor status, feeding status, and titers against diphtheria and tetanus vaccines was assessed using clinical information provided by the respective treating physicians and care providers. Unless otherwise indicated, "week" refers to weeks subsequent to ERT initiation.

Study approval. After discussion with the Northern California Regional Kaiser Genetic Diseases Treatment Advisory Board (Oakland, California, USA) and after obtaining written parental informed consent, a trial of a bortezomib-based regimen was commenced in Patient 1 . The parents of Patients 2 and 3 agreed to physician-directed use of bortezomib as a life-saving measure. Written informed consent have also been obtained for Duke IRB approved protocol Pro00001562.

\section{Author contributions}

Conception and design were by ZBK, SNP, and PSK. Development of methodology was performed by ZBK, SNP, and PSK. Acquisition of data was performed by ZBK, JAK, DV, CB, RG, DWS, and PSK. Analysis and interpretation of data were performed by ZBK, SNP, ASR, and PSK. Writing, review, and/or revision of the manuscript were done by ZBK, SNP, JAK, DV, CB, RG, DWS, PM, ASR, and PSK.

\section{Acknowledgments}

The authors thank the patients and their families who participated in this study, as well as the local physicians and clinical teams involved in their care. The authors would also like to thank Cynthia Meteyer for her help with coordinating the data transfer. This research was supported by a grant from Genzyme Corporation, a Sanofi Company (Cambridge, Massachusetts, USA) and in part by the Lysosomal Disease Network, a part of NIH Rare Diseases Clinical Research Network (RDCRN). The Lysosomal Disease Network (U54NS065768) is a part of the NIH RDCRN, supported through collaboration between the NIH Office of Rare Diseases Research (ORDR) at the National Center for Advancing Translational Science (NCATS), the National Institute of Neurological Disorders and Stroke (NINDS) and National Institute 
of Diabetes and Digestive and Kidney Diseases (NIDDK). The content is solely the responsibility of the authors and does not necessarily represent the official views of the NIH. The funding sources had no role in the study design, data collection and analysis, decision to publish, or preparation of the manuscript. The opinions in this article belong to the authors and do not necessarily represent the views and/or policies of the US FDA.

Address correspondence to: Priya S. Kishnani,Duke University Medical Center, 905 South LaSalle Street, GSRB1,Durham, North Carolina 27710 USA. Phone: 919.684.2036. E-mail: priya.kishnani@duke.edu.

1. Kishnani PS, et al. A retrospective, multinational, multicenter study on the natural history of infantile-onset Pompe disease. $J$ Pediatr. 2006;148(5):671-676.

2. van den Hout HM, et al. The natural course of infantile Pompe's disease: 20 original cases compared with 133 cases from the literature. Pediatrics. 2003;112(2):332-340.

3. Kishnani PS, et al. Recombinant human acid [alpha]-glucosidase: major clinical benefits in infantile-onset Pompe disease. Neurology. 2007;68(2):99-109.

4. Kishnani PS, et al. Early treatment with alglucosidase alpha prolongs long-term survival of infants with Pompe disease. Pediatr Res. 2009;66(3):329-335.

5. Nicolino M, et al. Clinical outcomes after long-term treatment with alglucosidase alfa in infants and children with advanced Pompe disease. Genet Med. 2009; 11(3):210-219.

6. Kishnani PS, et al. Cross-reactive immunologic material status affects treatment outcomes in Pompe disease infants. Mol Genet Metab. 2010;99(1):26-33.

7. Berrier KL, et al. CRIM-negative infantile Pompe disease: characterization of immune responses in patients treated with ERT monotherapy. Genet Med. 2015;17(11):912-918.

8. Banugaria SG, et al. The impact of antibodies on clinical outcomes in diseases treated with therapeutic protein: lessons learned from infantile Pompe disease. Genet Med. 2011;13(8):729-736.

9. Hobson-Webb LD, et al. Autopsy findings in late-onset Pompe disease: a case report and systematic review of the literature. Mol Genet Metab. 2012;106(4):462-469.

10. Banugaria SG, et al. Algorithm for the early diagnosis and treatment of patients with cross reactive immunologic material-negative classic infantile pompe disease: a step towards improving the efficacy of ERT. PLoS One. 2013;8(6):e67052.

11. Banugaria SG, Patel TT, Kishnani PS. Immune modulation in Pompe disease treated with enzyme replacement therapy. Expert Rev Clin Immunol. 2012;8(6):497-499.

12. Messinger YH, et al. Successful immune tolerance induction to enzyme replacement therapy in CRIM-negative infantile Pompe disease. Genet Med. 2012;14(1):135-142.

13. Elder ME, et al. B-Cell depletion and immunomodulation before initiation of enzyme replacement therapy blocks the immune response to acid alpha-glucosidase in infantile-onset Pompe disease. J Pediatr. 2013;163(3):847-854.e1.

14. Hunley TE, et al. Nephrotic syndrome complicating alpha-glucosidase replacement therapy for Pompe disease. Pediatrics. 2004; 114(4):e532-535.

15. Deodato F, et al. Immune tolerance induced using plasma exchange and rituximab in an infantile Pompe disease patient. $J C h i l d$ Neurol. 2014;-29(6):850-854.

16. Banugaria SG, et al. Bortezomib in the rapid reduction of high sustained antibody titers in disorders treated with therapeutic protein: lessons learned from Pompe disease. Genet Med. 2013;15(2):123-131.

17. Myozyme, [Package Insert]. 2006, Genzyme Corporation: Cambridge (MA)

18. Vogel M, Staller W, Buhlmeyer K. Left ventricular myocardial mass determined by cross-sectional echocardiography in normal newborns, infants, and children. Pediatr Cardiol. 1991;12(3):143-149.

19. Young SP, et al. Long-term monitoring of patients with infantile-onset Pompe disease on enzyme replacement therapy using a urinary glucose tetrasaccharide biomarker. Genet Med. 2009;11(7):536-541.

20. Mendelsohn NJ, et al. Elimination of antibodies to recombinant enzyme in Pompe's disease. N Engl J Med. 2009;360(2):194-195.

21. Richardson PG, et al. Bortezomib or high-dose dexamethasone for relapsed multiple myeloma. $N$ Engl J Med. 2005;352(24):2487-2498.

22. Kim KH, Messinger YH, Burton BK. Successful reduction of high-sustained anti-idursulfase antibody titers by immune modulation therapy in a patient with severe mucopolysaccharidosis type II. Mol Genet Metab Rep. 2015;2:20-24.

23. Kishnani PS, et al. Chinese hamster ovary cell-derived recombinant human acid alpha-glucosidase in infantile-onset Pompe disease. J Pediatr. 2006;149(1):89-97.

24. Bali DS, et al. Predicting cross-reactive immunological material (CRIM) status in Pompe disease using GAA mutations: lessons learned from 10 years of clinical laboratory testing experience. Am J Med Genet C Semin Med Genet. 2012;160C(1):40-49. 